\title{
Alinhamento de Diferentes Projetos Pedagógicos de Cursos de Medicina com as Diretrizes Curriculares Nacionais
}

\section{Alignment of Different Pedagogical Projects of Medicine Courses with the National Curricular Guidelines}

\author{
Carlos Alberto de Oliveira ${ }^{I}$ \\ Maria Helena Senger \\ Oscarina da Silva Ezequiel ${ }^{I I}$ \\ Eliana Amaral ${ }^{I I I}$
}

\section{PALAVRAS-CHAVE}

- Curriculum

- Educação Médica.

- Metodologias Ativas.

- Aprendizagem Baseada em Problemas.
${ }^{I}$ Pontifícia Universidade Católica de São Paulo, São Paulo, São Paulo, Brasil.

"Iniversidade Federal de Juiz de Fora, Juiz de Fora, Minas Gerais, Brasil.

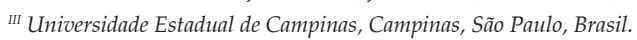

O objetivo desta pesquisa foi analisar os projetos pedagógicos de dois cursos (PPC) de graduação em Medicina para determinar o grau de alinhamento às Diretrizes Curriculares Nacionais (DCN) do ensino médico, visando compreender as diferenças decorrentes dos modelos curriculares adotados. Três especialistas em ensino médico foram convidados a analisar os documentos relativos aos projetos pedagógicos de curso (PPC) e preencher um instrumento de avaliação desenvolvido para avaliar adesão às DCN 2001, previamente publicado, com dez dimensões. O escore calculado por meio do instrumento classifica as escolas em relação ao grau de aderência às $D C N$, sendo valores de escore de $0 a \leq 20 \%$, de $20 a \leq 40 \%$, de $40 a \leq 60 \%$, de $60 a \leq 80 \%$ e de $>80 \%$ considerados, respectivamente, divergente, fracamente aderente, regular aderência, aderente moderadamente e fortemente aderente. Também se realizou análise de conteúdo dos PPC para buscar evidências sobre os domínios no texto. Os escores totais, de 47,5\% (IES1) e 82,5\% (IES2), mostraram adesão regular do PPC da IES1 e acentuada do PPC da IES2 às DCN 2001. Essas diferenças se manifestaram especialmente nas dimensões da adequação da metodologia de ensino à concepção do curso, da inter-relação das disciplinas na concepção e execução do currículo e na existência de módulos ou temas integradores. As análises dos PPC mostraram que, de maneira geral, há aderência às DCN, em diferentes estágios. Observou-se boa confiabilidade desse instrumento utilizado na análise dos três avaliadores para ambas as instituições de ensino superior (alfa de Cronbach de 0,77 para a IES1 e de 0,75 para a IES2). A comparação das médias dos escores dos avaliadores por dimensão foi submetida a uma análise de significância com o Teste t de Student. Conclui-se que as duas escolas, em intensidade e por caminhos diferentes e respeitando a cultura institucional, incorporaram paulatinamente o arcabouço das diretrizes curriculares nacionais. 


\section{KEY-WORDS}

- Curriculum.

- Medical Education.

- Active Methodologies.

- Problem-Based Learning.

Recebido em: 26/9/18

Aceito em: 17/12/18
ABSTRACT

The objective of this research was to analyze the pedagogical projects of two undergraduate medical education courses (CPP) to determine the degree of alignment with the National Curricular Guidelines (NDC) of Medical Education, aiming to understand the differences that arise from the curricular models adopted. Three medical education experts were invited to review the documents relating to the course pedagogical projects (CPP) and to complete an evaluation instrument developed to evaluate adherence to the previously published ten-dimensional DCN 2001. The score calculated from the instrument classifies the schools in relation to the degree of adherence to the DCN, with scores ranging from $0 \leq$ to $20 \%$, from 20 to $\leq 40 \%$; 40 to $\leq$ to $60 \%$; from 60 to $\leq$ to $80 \%$ and from > $80 \%$ considered respectively, divergent, poorly adherent, regular adherence, adherent moderately and strongly adherent. Content analysis of PPPS was also conducted to search for evidence on domains in the text. The total scores of $47.5 \%$ (Institution of Higher Education 1) and $82.5 \%$ (Institution of Higher Education 2), showed regular adherence of the PPC of Institution of Higher Education 1 and marked of the CPP of Institution of Higher Education 2 to DCN 2001. These differences were manifested especially in the dimensions of the adequacy of the teaching methodology to the course design, of the interrelationship of the disciplines in the conception and execution of the curriculum and in the existence of integrating modules or themes. The CPP analyzes showed that, in general, there is adherence to the DCN in different stages. We observed a good reliability of this instrument used in the analysis of the three evaluators for both institutions of higher education (Cronbach's alpha of 0.77 for Institution of Higher Education 1 and Cronbach's alpha of 0.75 for the Institution of Higher Education 2). The comparison of the means of the scores of the evaluators by size was submitted to a significance analysis with the Student $t$ Test. It is concluded that the two schools, in intensity and in different ways and respecting the institutional culture, gradually incorporated the framework of the national curricular guidelines.

\section{INTRODUÇÃO}

As Diretrizes Curriculares Nacionais (DCN) homologadas em $2014^{1,2}$ reforçaram a recomendação das DCN de $2001^{1}$ de que os cursos de Medicina utilizem metodologias ativas de ensino-aprendizagem. Ambas as diretrizes apontam que os currículos devem contemplar o perfil acadêmico e profissional, as habilidades, as competências e os conteúdos para que o estudante aprenda a aprender, facilitando seu engajamento num processo de educação permanente. Como resultado, os estudantes deveriam demonstrar comunicação efetiva, facilidade para tomada de decisão e de liderança, além de ter domínio dos conhecimentos científicos básicos de natureza biopsicossocioambiental, utilizando-se de recursos propedêuticos para exercer uma medicina baseada em evidências científicas, com senso de responsabilidade social e compromisso com a cidadania. Já em $2001^{1}$ se afirmava que os processos avaliativos deveriam se basear em competências, habilidades e atitudes. Ainda, as DCN de 2014 afirmam, em seu Artigo 7º, que o graduando de- verá estar apto a se corresponsabilizar por sua formação inicial e continuada para conquistar autonomia intelectual, além de demonstrar responsabilidade social e compromisso com a formação das futuras gerações de profissionais de saúde ${ }^{2}$. Assim, o curso de Medicina exige um projeto pedagógico diferenciado, no qual o professor deve ser um facilitador ou moderador, mediando o processo de ensino-aprendizagem ${ }^{1,3}$.

Os métodos centrados no estudante, frequentemente chamados de metodologias ativas de ensino-aprendizagem ${ }^{4}$, caracterizam-se por buscar a integração de saberes com atitudes pautadas na reflexão sobre a prática, contribuindo para o desenvolvimento de competências ${ }^{5,6}$. Como principais características desses métodos destacam-se a promoção de proatividade comprometimento do aluno no processo, vinculação da aprendizagem à realidade e à capacidade para nela intervir, além da colaboração e cooperação entre pares ${ }^{7,8}$. Ainda, os egressos das escolas médicas que utilizam metodologias centradas no estudante deveriam demonstrar foco de atuação no cuidado. 
Em meta-análise de 2017, concluiu-se que não houve diferença considerável em desempenho cognitivo entre currículos que utilizaram Aprendizagem Baseada em Problemas (ABP) e baseados em conferências; no entanto, a ABP influenciou positivamente atividades mais voltadas a laboratório e habilidades sociais ${ }^{9}$. Schauber et al. ${ }^{10}$, num estudo com 1.646 estudantes, concluíram que a forma como o estudante aborda seu processo de aprendizagem - o que inclui aspectos acerca de sua percepção do ambiente educacional e confiança em sua autoeficácia e capacidade de autorregulação da aprendizagem - é o maior diferencial de desempenho.

As instituições de ensino superior (IES) precisam de tempo para modificar seus projetos pedagógicos e suas práticas. Os cursos de Medicina encontram dificuldades para adequar sua metodologia a processos mais centrados no estudante, especialmente em seus anos iniciais, com resistências à mudança por parte de professores e alunos ${ }^{9-15,16}$. Independentemente do PPC e das práticas pedagógicas em curso, há uma demanda legal para seguir as recomendações das DCN. Assim, conhecer o alinhamento entre os objetivos de formação nos PPC e as competências preconizadas pelas DCN 2001 pode contribuir para identificar facilitadores da implantação de marcos legais em instituições com características similares, acelerando as mudanças demandadas para a formação de melhores profissionais. Este estudo tem por objetivo analisar os projetos pedagógicos de dois cursos de Medicina quanto às suas propostas em relação às recomendações das DCN 2001, identificando as mudanças já implantadas.

\section{MÉTODOS}

Realizou-se uma avaliação por análise documental dos projetos pedagógicos de curso (PPC) de Medicina em contraste com um instrumento específico, que permite avaliar a adesão dos PPC às DCN 2001. O objetivo incluiu extrair dos PPC evidências de convergência ou divergência em relação às recomendações das DCN 2001, avaliando as dez dimensões de um instrumento próprio para este fim, descrito na literatura por Haddad et al. ${ }^{17}$.

Esta pesquisa foi realizada de junho de 2017 a junho de 2018, envolvendo duas escolas médicas: uma instituição confessional (IES2) e outra de uma autarquia municipal (IES 1), ambas do Estado de São Paulo. A IES 1, CI $=4$ e IGC $=4^{25}$, disponibiliza 120 vagas para a graduação em Medicina, cujo currículo é organizado no regime seriado, por disciplina, com integralização mínima de seis anos e máxima de nove anos. A carga horária total do curso é de 10.310 horas, sendo 5.920 horas cumpridas do primeiro ao quarto ano, 3.600 horas de internato e 790 horas de atividades complementares. Está loca- lizada no Estado de São Paulo numa cidade com cerca de 400 mil habitantes. A IES 2, CI $=4$ e IGC $=4^{25}$, também localizada no Estado de São Paulo, numa cidade com cerca de 650 mil habitantes, disponibiliza 130 vagas para o curso de Medicina. $\mathrm{O}$ curso utiliza metodologia de ensino predominantemente ABP, com o currículo desenvolvido segundo eixos norteadores. Tem um período de integralização mínima de seis anos e máxima de nove anos. A carga horária total é de 7.532 horas, desenvolvidas em 3.360 horas do primeiro ao terceiro ano, 4.072 horas no internato do quarto ao sexto ano e cem horas de atividades complementares (atividades acadêmico-científico-culturais).

Três avaliadores (pesquisador principal e dois pesquisadores auxiliares) fizeram a leitura dos projetos pedagógicos e os analisaram com base em instrumento composto por dez dimensões, após treinamento no uso do mesmo ${ }^{2}$. De forma individual, cada avaliador determinou o escore de cada IES, que variou de 10 a 50 pontos, após analisar cada uma das dimensões, atribuindo valor a cada item: muito fraco (1), fraco (2), regular (3), bom (4) e muito bom (5).

A avaliação final de cada IES foi obtida pela média do somatório dos escores dos três avaliadores. Utilizamos um índice, adaptado de Haddad et al. ${ }^{2}$, que denominamos $\mathrm{I}_{\mathrm{c} 10}$ - índice completo das dez dimensões, definido como a razão composta pela diferença entre a média do somatório dos escores dos três juízes e o escore mínimo possível (10) e a diferença entre o escore máximo e o escore mínimo possível (40), multiplicado por 100. Desta forma, obtêm-se o valor em percentagem.

$I_{c 10=}\left\{\left[\right.\right.$ média dos escores ${ }^{*}-\left(\right.$ escore mín. $\left.\left.{ }^{* *}\right)\right] /[$ (escore máximo $\left.^{* * *}\right)-\left(\right.$ escore mín. $\left.{ }^{* *}\right)$ ]\} X 100

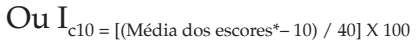

* Escore médio obtido pela IES (varia de 10 a 50 pontos) - após análise dos juízes.

** Escore mínimo possível (10 pontos).

*** Escore máximo possível (50 pontos).

Com o valor do índice expresso em percentagem, criamos a seguinte classificação proporcional: se o índice apresentava percentagens de 0 a $\leq 20 \%$, o PPC do curso tinha divergência em relação às DCN-EM; de $20 \mathrm{a} \leq 40 \%$, havia aderência muito fraca às DCN-EM; de $40 \mathrm{a} \leq 60 \%$, o PPC do curso tinha aderência regular às DCN-EM; se o índice estivesse na faixa de 60 $\mathrm{a} \leq 80 \%$, o PPC do curso era aderente às DCN-EM; e se estivesse acima de $80 \%$, o PPC do curso era fortemente aderente às DCN-EM

Os dados quantitativos foram digitados no Excel e analisados no Statistical Package for the Social Sciences (SPSS) v. 24.0. Realizou-se análise de confiabilidade entre os avaliadores com o alfa de Cronbach ${ }^{18-21}$ e diferença das médias entre IES1 e IES2 utilizando-se o Teste $\mathrm{t}^{22}$ com nível de significância $\mathrm{p}<0,05$. 
Na etapa qualitativa da pesquisa, foi realizada análise dos conteúdos dos PPC, buscando identificar trechos que evidenciassem a situação da IES em relação aos domínios presentes no questionário.

Este estudo foi aprovado pela Comitê de Ética em Pesquisa (CEP) da IES2 sob o número CAAE 62459816.5.0000.5373 e pelo CEP da IES1 sob o número CAAE 62459816.5.3001.5412, após aprovação nas respectivas coordenações de graduação.

\section{RESULTADOS}

Os índices atribuídos à IES1 mostraram aderência regular às DCN 2001 (Ic10 = 47,5\%) e a IES2 se mostrou fortemente aderente $(\mathrm{Ic} 10=82,5 \%)$ (Tabela 1$)$. Foram observadas diferenças estatisticamente significativas entre as escolas nas dimensões de coerência do currículo com o perfil profissional, adequação da metodologia de ensino, inter-relação das disciplinas na concepção e execução do currículo, coerência do sistema de avaliação com a concepção do curso, existência de módulos ou temas integradores, existência de atividades formativas de prática profissional ao longo do curso, constituição e dimensão do treinamento obrigatório, existência de rede de comunicação científica e prática nos serviços de saúde dos três níveis de atenção, mas não se observou na dimensão de laboratório (Tabela 2). As avaliações entre os três avaliadores apresentaram confiabilidade de 0,77 para IES1 (alfa de Cronbach) e de 0,75 para IES 2.

\begin{tabular}{|c|c|c|c|c|c|c|}
\hline DCNEM & PPC IES 1 & PPC IES 1 & PPC IES 1 & PPC IES 2 & PPC IES 2 & PPC IES 2 \\
\hline Dimensões & Avaliador 1 & Avaliador 2 & Avaliador 3 & Avaliador 1 & Avaliador 2 & Avaliador 3 \\
\hline 1. Coerência do currículo com o perfil desejado do egresso & 2 & 3 & 3 & 4 & 5 & 5 \\
\hline 2. Adequação da metodologia de ensino à concepção do curso & 2 & 2 & 2 & 5 & 5 & 5 \\
\hline $\begin{array}{l}\text { 3. Inter-relação das disciplinas na concepção e execução do } \\
\text { currículo }\end{array}$ & 2 & 3 & 4 & 5 & 5 & 4 \\
\hline $\begin{array}{l}\text { 4. Coerência do sistema de avaliação do processo ensino- } \\
\text { aprendizagem com a concepção do curso }\end{array}$ & 3 & 2 & 3 & 4 & 5 & 4 \\
\hline 5. Existência de módulos ou temas integradores & 2 & 2 & 2 & 5 & 5 & 5 \\
\hline $\begin{array}{l}\text { 6. Existência de atividades formativas de prática profissional } \\
\text { ao longo do curso }\end{array}$ & 4 & 3 & 4 & 4 & 4 & 4 \\
\hline 7. Constituição e dimensão de áreas de treinamento obrigatório & 3 & 4 & 3 & 4 & 5 & 4 \\
\hline 8. Existência de rede de comunicação científica & 3 & 3 & 3 & 4 & 2 & 3 \\
\hline 9. Serviços de Unidades de Saúde dos três níveis & 4 & 3 & 3 & 4 & 5 & 5 \\
\hline 10. Serviços de laboratórios de ensino & 3 & 4 & 3 & 3 & 4 & 3 \\
\hline Escore total & 28 & 29 & 30 & 42 & 45 & 42 \\
\hline
\end{tabular}

* Média da avaliação dos três juízes para IES 1 = 29 e para a IES $2=43$.

Fonte: Autor.

Escore da IES 1:

$I_{c 10}($ IES 1) $=$

Escore da IES 2:

$I_{c 10}($ IES 1) $=\{[43-10] /[50-10] / \times 100=82,5 \%$

\section{TABELA 2}

Média e desvio-padrão de cada dimensão para as duas escolas estudadas: IES1 e IES2

Dimensões

(1)

IES1

IES 2

1. Coerência do currículo com o perfil

\begin{tabular}{cccccc} 
Média & DP* & \multicolumn{2}{c}{ Média } & DP* & P** \\
2,00 & 0,00 & 4,66 & 0,47 & $<\mathbf{0 , 0 0 1}$ \\
2,00 & 0,00 & 5,00 & 0,00 & $<\mathbf{0 , 0 0 1}$ \\
2,66 & 0,47 & 4,66 & 0,47 & $<\mathbf{0 , 0 1}$ \\
2,66 & 0,47 & 4,33 & 0,47 & $<\mathbf{0 , 0 1}$ \\
2,00 & 0,00 & 5,00 & 0,00 & $<\mathbf{0 , 0 0 1}$ \\
3,33 & 0,47 & 4,00 & 0,00 & $\mathbf{0 , 0 3}$ \\
3,33 & 0,47 & 4,33 & 0,47 & $\mathbf{0 , 0 3}$ \\
2,66 & 0,47 & 4,00 & 0,82 & $\mathbf{0 , 0 4}$ \\
3,00 & 0,00 & 4,66 & 0,47 & $<\mathbf{0 , 0 1}$ \\
3,33 & 0,47 & 3,33 & 0,47 & 0,50
\end{tabular}

2. Adequação da metodologia de ensino

3. Inter-relação das disciplinas na concepção e execução do currículo

4. Coerência do sistema de avaliação do processo ensino-aprendizagem com a concepção do curso

5. Existência de módulos ou temas integradores

6. Existência de atividades formativas de prática profissional ao longo do curso

7. Constituição e dimensão de áreas de treinamento obrigatório

8. Existência de rede de comunicação científica

9. Serviços de Unidades de Saúde dos três níveis

3,33

*DP-desvio-padrão e ** $P$ - teste $t$.

Fonte: Autor. 
A análise do discurso dos textos dos projetos políticos pedagógicos de cada escola encontra-se no Quadro 1. Chama a atenção a diferença entre as notas atribuídas às dimensões coerência do currículo com o perfil desejado do egresso, ade- quação da metodologia de ensino à concepção do curso, inter-relação das disciplinas na concepção e execução do currículo, existência de módulos ou temas integradores e serviços de unidades de saúde dos três níveis.

\begin{tabular}{|c|c|}
\hline & $\begin{array}{l}\text { QUADro } 1 \\
\text { Exemplos de conteúdo das dimensõ }\end{array}$ \\
\hline Dimensões & IES 1 \\
\hline $\begin{array}{l}\text { 1. Coerência do currículo } \\
\text { com o perfil desejado do } \\
\text { egresso }\end{array}$ & $\begin{array}{l}\text { [...] ao formar um profissional que, além de dominar os } \\
\text { princípios básicos da medicina curativa, domina também } \\
\text { os princípios de promoção da saúde coletiva, o que implica } \\
\text { na importância de saber atuar frente às necessidades da } \\
\text { comunidade. } \\
\text { No ano de } 2014 \text { o quadro curricular passou por adequações, } \\
\text { tendo em vista nova ampliação dos cenários de prática } \\
\text { para os alunos desta IES junto aos serviços vinculados à } \\
\text { Secretaria de Saúde do Município, especialmente no que se } \\
\text { refere à atenção básica. (p. 32-32) }\end{array}$ \\
\hline
\end{tabular}

2. Adequação da metodologia de ensino à concepção do curso

3. Inter-relação das disciplinas na concepção e execução do currículo

4. Coerência do sistema de avaliação do processo ensino-aprendizagem com a concepção do curso

5. Existência de módulos ou temas integradores
[...] durante os dois primeiros anos da formação médica, é essencial a organização do ensino denominado básico. As atividades do Internato desenvolvem-se [...] basicamente em estágios hospitalares, ambulatoriais e na rede básica de saúde da Secretaria Municipal de Saúde do Município. ( $p$. 47-51)

No $1^{\circ}$ e no $2^{\circ}$ ano do curso há disciplinas anuais e semestrais [...] No $3^{\circ}$ e no $4^{\circ}$ ano as disciplinas também são anuais ou semestrais [...] no $5^{\circ}$ e no $6^{\circ}$ anos [...] passam por um período em cada estágio. ( $p$. 47-51)

Conforme o Regimento Geral desta IES, a avaliação da aprendizagem é realizada em cada disciplina, abrangendo todos os aspectos de aproveitamento, mediante procedimentos específicos e atividades curriculares, tais como: provas, pesquisas, arguições, trabalhos práticos em laboratórios e campo, seminários e estágios, entre outras. ( $p$. 83-85)

\section{Entende-se que a discussão e a problematização} interdisciplinar devem estar presentes desde o início da vida acadêmica do estudante, mesmo reconhecendo que a atual organização curricular, algumas vezes, ainda dificulte a operacionalização da interdisciplinaridade. (p. 48-49)

IES 2

[...] Priorizar a educação problematizadora em detrimento da tradicional; centralizar o ensino nas necessidades do aluno; garantir o contato do aluno de medicina, desde o primeiro ano da faculdade, com as realidades de saúde e socioeconômicas da comunidade; realizar um processo educativo interativo e construtivo; individualizar a educação; realizar avaliação diferenciada, integrada e integral. ( $p$. 16-17)

[...] grande reforma curricular que culminou com a mudança para metodologia ativa.

As competências e habilidades a serem adquiridas pelo estudante nos doze semestres do curso médico serão desenvolvidas por meio das diversas estratégias pedagógicas articuladas em cada ano do curso de graduação. (p. 18)

[...] Assim, um currículo organizado por módulos pode oferecer essas possibilidades, permitindo diferentes combinações de conhecimentos disciplinares e de aplicações. (p. 20)

São utilizadas diversas formas de avaliação dos estudantes, em consonância com o que é praticado nos anos anteriores. São realizadas:

Avaliação formativa (tutoria, portfólio, mini-CEX, prova de progresso, apresentação e caso clínico);

Avaliação somativa (salto triplo, prova em estações, prova escrita, mini-CEX, relatórios finais). (p. 84-87)

[...] definem-se módulos como organizações didático/pedagógicas que se caracterizam por atividades interdisciplinares que buscam desenvolver competências através da inter-relação de conceitos e organização de atividades. A construção curricular modulada favorece a vivência de aprendizagens relacionais, ou seja, aprendizagens que se articulem com temas transversais de modo a se dinamizar num movimento em espiral, onde temas, proposições, problemáticas, conceitos fundantes da experiência formativa sejam vivenciados perpassando toda a formação e sendo perpassados pelos conhecimentos específicos dessa mesma formação. (p. 24-25)

6. Existência de atividades formativas de prática profissional ao longo do curso
O Internato constitui o último núcleo do curso de graduação médica, com duração de dois anos. No internato a formação teórico-prática é intensiva e contínua, sob a orientação científica e didática do corpo docente da faculdade, prioritariamente, e do corpo clínico das Instituições de Saúde conveniadas com a faculdade. (p. 50)
Trazer aspectos da teoria como suporte aos conhecimentos médicos para a prática é possível com o contato direto com os pacientes e usuários do sistema de saúde. A melhor forma para propiciar tal articulação se dá por meio da inserção precoce do estudante de medicina, ou seja, desde o primeiro instante no Curso de Medicina os estudantes são levados aos cenários de prática que lhes possibilitam o contato com os usuários do sistema de saúde, pacientes, seus núcleos familiares e ambiente em que vivem. Ao longo do curso e, especialmente, durante o internato, agora estendido para os últimos três anos do curso, há a possibilidade de que o diálogo entre teoria e prática se faça de forma efetiva. ( $p .22-24)$ 
(continuação)

\begin{tabular}{ll}
\hline Dimensões & IES 1 \\
\hline $\begin{array}{l}\text { 7. Constituição e } \\
\text { dimensão de áreas de } \\
\text { treinamento obrigatório }\end{array}$ & $\begin{array}{l}\text { áreas já citadas, um núcleo de estágio em Ambulatórios, } \\
\text { pertencentes à rede de atenção básica do município e um } \\
\text { estágio optativo, realizado em instituições renomadas, por } \\
\text { meio de parcerias firmadas com esta IES. }(p .51)\end{array}$
\end{tabular}

8. Existência de rede de comunicação científica

9. Serviços de unidades de saúde dos três níveis

10. Serviços de laboratórios de ensino
Desde 1976, esta IES edita a Revista Perspectivas Médicas. A partir do ano de 1995, a revista foi atualizada e reestruturada, com publicações de periodicidade anual, sendo editada e publicada ininterruptamente por 16 anos. Em 2013 obteve B3 no Qualis Capes e recebeu pelo International Index Copernicus Value o fator de impacto de $5,15$, passando para a periodicidade quadrimestral. ( $p .79)$

\section{IES 2}

Na presente proposta estão incluídas situações do cotidiano em que há convivência entre professores e preceptores médicos e não-médicos. Isso ocorre especialmente nas atuações em que os estudantes estão na prática de aprendizagem das Unidades Básicas de Saúde (atividades com profissionais da Antropologia), nos Projetos Terapêuticos Singulares (Enfermeiros) e em atividades específicas no Internato (Saúde Coletiva, Cirurgia e Obstetrícia).

Nos três últimos anos são desenvolvidas as atividades eminentemente práticas do Internato, que correspondem ao estágio curricular obrigatório de treinamento em serviço do curso. ( $p .30)$

Livre acesso ao acervo, possibilitando ao usuário o manuseio das obras; Acessibilidade para portadores de necessidades especiais; Empréstimo domiciliar; Página web da biblioteca e Catálogo on-line (Lumen); Serviços on-line de reserva e renovação de empréstimos; Serviços de Pesquisa e levantamento bibliográfico; Serviços de Recuperação de documentos; Comutação bibliográfica (SCAD e Comut);

Capacitação para Pesquisa (Fontes de Informação, Gerenciadores de Referência);

Orientação e normalização de trabalhos acadêmicos; Acesso a importantes fontes de informação on-line; Biblioteca digital institucional de Teses e Dissertações; Portal de Revistas Publicadas pela Instituição; Suporte técnico à editoração de revistas eletrônicas publicadas no Campus Sorocaba; Suporte à utilização de ambiente Moodle para apoio ao ensino presencial; Suporte ao desenvolvimento de conteúdos pedagógicos. (p. 105-106)

[...] a prática constitui uma das dimensões para a produção de conhecimentos; para tanto, desde o primeiro semestre o aluno terá contato com as atividades hospitalares, seja no momento de Habilidades Médicas (4 horas/aula), seja na Prática de Atenção à Saúde (4 horas/aula). Com o desenvolver do curso, no 5 e 6 semestre, as atividades de Habilidades duplicam. No internato, a integração entre teoria e prática é total. (p. 30)

Biotério, Lab. de Anatomia, Lab. de Anatomia Patológica, Lab. de Biologia Celular, Lab. de Biomateriais, Lab. de Bioquímica, Lab. de Farmacologia, Lab. de Fisiologia, Lab. de Habilidades Clínicas, Lab. de Histologia, Lab. de Imunologia, Lab. de Parasitologia, Lab. Morfofuncional, Lab. de Microbiologia, Lab. de Simulação de Procedimentos, Lab. de Técnicas Cirúrgicas. (p. 103)

\section{DISCUSSÃO}

Nossos resultados evidenciaram que há uma diferença de alinhamento dos projetos pedagógicos do curso de Medicina das instituições às DCN 2001, a qual se manifesta em todas as dimensões curriculares, dos serviços de saúde nos três níveis e da infraestrutura, exceto na estrutura de laboratórios.

As diretrizes de 2001, que recomendam a formação global do médico e embasaram mudanças curriculares nas escolas de Medicina do País, foram fruto de ampla discussão nos anos 1990, da qual participaram diversas entidades interessadas como a Associação Brasileira de Educação Médica (Abem), a Direção Executiva Nacional dos Estudantes de Medicina (Denem) e o Conselho Federal de Medicina (CFM) ${ }^{23}$, entre outras.
Pelas DCN, os cursos de saúde devem ter abordagem pedagógica centrada no estudante, favorecer a articulação de conhecimentos e o trabalho em equipes multiprofissionais, ampliando os cenários das práticas de atenção à saúde. Os estudantes devem aprender a aprender, para aprender a ser, aprender a fazer e, com autonomia e discernimento, estar capacitados para assegurar a integralidade no atendimento dos indivíduos, das famílias e das comunidades ${ }^{24}$. Isto reforça o conceito de que o currículo é "vivo", dinâmico e deve decorrer de uma construção coletiva e multifacetada, com a contribuição dos diversos indivíduos.

Ainda que as DCN descrevam as competências esperadas em múltiplos domínios, cada escola deve encontrar sua 
trajetória, desenhada pelo projeto pedagógico de curso. Este projeto deve orientar docentes, discentes, preceptores e colaboradores administrativos em relação ao direcionamento da formação, que engloba aspectos de humanismo e profissionalismo, além dos conhecimentos e habilidades técnicas.

As DCN passaram a ter força de lei quando a versão 2014 foi publicada, sendo seus princípios incluídos nos instrumentos de acreditação e reconhecimento ou renovação e reconhecimento dos cursos de Medicina². O alinhamento às DCN é um dos itens de destaque no processo de avaliação dos cursos no Brasil, apoiado em instrumentos requeridos pelo Instituto Nacional de Avaliação (Inep) ${ }^{25}$. Documentos similares às DCN para a Medicina são encontrados em outros países, sob a responsabilidade de diferentes entidades governamentais, e orientam processos de acreditação: nos Estados Unidos, o Liaison Committee on Medical Education (LCME); no Canadá, o Outcome Project; no Reino Unido, o Tomorrow's Doctor, do General Medical Council (GMC), entre outros ${ }^{26-30}$.

Estudamos o alinhamento às DCN 2001, cujos princípios se assemelham aos das DCN 2014, porque estas últimas são muito recentes para melhor apreciação de sua implantação. A proposta de analisarmos a ocorrência de alinhamentos de dois projetos pedagógicos às diretrizes curriculares nacionais de 2001 teve o objetivo de fornecer dados para a discussão sobre as dificuldades de adoção das recomendações. A utilização de um instrumento que permita avaliar a adesão às DCN pode permitir, às escolas médicas, refletir sobre diferentes domínios relevantes para a formação geral do médico, em acordo com as recomendações dos arcabouços legais. Esse exercício, se adotado pela própria escola num mecanismo de autorreflexão, deve contribuir para acelerar os avanços necessários.

Acreditamos que o instrumento adotado neste estudo pode ser utilizado pelas escolas médicas para promover uma reflexão coletiva com vistas ao avanço nas propostas de formação do médico diante das recomendações definidas pelas DCN de 2001. No entanto, também serviriam para as DCN de 2014, visto que as dimensões escolhidas para a avaliação estão também contempladas nestas últimas. No entanto, as dez dimensões propostas no instrumento parecem insuficientes para identificar diferenças significativas na adesão às DCN. Para uma análise mais detalhada, são necessários outros estudos qualitativos.

Cabe destacar que as DCN não devem ser vistas como uma receita. Devem-se buscar caminhos alicerçados na cultura de cada instituição quando da implementação ou alteração dos projetos pedagógicos, adotando propostas críticas e inovadoras, refletidas pela sua comunidade. Independentemente do projeto pedagógico analisado, foi possível observar avan- ços na incorporação das principais recomendações apresentadas nas DCN, fundamentadas numa aprendizagem centrada no estudante, no sistema de saúde e que busque desenvolver competências de empatia, trabalho em equipe e educação permanente, em paralelo às demandas de formação técnico-profissional como médico.

Optamos por estudar escolas selecionadas de forma intencional, com desempenho avaliado pelo Exame Nacional de Estudantes (Enade), Índice Geral de Cursos idêntico (IGC - Sinaes) e tempo de início de atividades similar, ambas no Estado de São Paulo, que adotam processos educacionais diferenciados. As escolas selecionadas demonstraram receptividade à proposta, sendo que uma adotou currículo que utiliza ABP e não se organiza em departamentos e a outra mantém estrutura departamental e usa conferências predominantemente, associadas a ensino clínico.

\section{CONCLUSÃO}

Concluiu-se que as duas escolas, em momentos e de maneiras diferentes, seja qual for o modelo educacional adotado, incorporaram o arcabouço das diretrizes curriculares nacionais gradativamente em seus projetos pedagógicos.

A percepção diferenciada quanto ao alinhamento do PPC às DCN nas duas IES talvez decorra do fato de que a mudança na IES 2 ocorreu há mais tempo, e sabemos que toda mudança curricular requer tempo para ser internalizada e efetivamente incorporada por todos os colaboradores, docentes e discentes.

Ao propor mudanças curriculares, é necessário observar que buscar a convergência com as DCN vai muito além da flexibilidade, da interdisciplinaridade e da articulação teórica e prática. Acima de tudo, é fundamental observar o conteúdo obrigatório, a distribuição da carga horária entre os núcleos de formação geral ou básica e profissional ou clínica, as atividades complementares e as atividades desenvolvidas no campo profissional, seja em ambiente hospitalar ou nas Unidades Básicas de Saúde.

\section{AGRADECIMENTOS}

Os autores agradecem a colaboração das coordenações dos cursos de Medicina da IES1 e da IES2 e das assessorias pedagógicas dos cursos de Medicina analisados, por disponibilizarem os projetos pedagógicos dos cursos para a análise documental, assim como ao Prof. Dr. Reinaldo Gianini (PUC-SP) pelas análises estatísticas. 


\section{REFERÊNCIAS}

1. Brasil. Ministério da Educação. Conselho Nacional de Educação. Câmara de Educação Superior. Resolução CNE/CES n ${ }^{\circ} 4$, de 7 de novembro de 2001. Diário Of da União. 2001;(9 nov):38.

2. Brasil. Ministério da Educação. Conselho Nacional de Educação Câmara de Educação Superior. Resolução CNE/ CES no 3, de 20/06/2014. Diretrizes Curriculares Nacionais do Curso de Graduação em Medicina. Diário Oficial da União. 2014;8-11.

3. Mitre SM, Siqueira-Batista R, Girardi-de-Mendonça JM, Morais-Pinto NM de, Meirelles C de AB, Pinto-Porto C, et al. Metodologias ativas de ensino-aprendizagem na formação profissional em saúde: debates atuais. Cien Saude Colet. 2008;13(suppl 2):2133-44.

4. Almeida MTC, Batista NA. Ser docente em métodos ativos de ensino-aprendizagem na formação do médico. Revista Brasileira de Educação Médica 2011 p. 468-76.

5. Batista NA, Batista SH, Goldenberg P, Seiffert O, Sonzogno MC. O enfoque problematizador na formação de profissionais da saúde. Rev Saude Publica. 2005;39(2):231-7.

6. Berbel NAN, Gamboa SAS. A metodologia da problematização com o Arco de Maguerez: uma perspectiva teórica e epistemológica. Filos e Educ. 2012;3(2):264-87.

7. Bonwell CC, Eison JA, Eric S, Education H, Dc W, Digest CE. Active learning: creating excitement in the classroom. Higher Education. Washington: Wiley; 1991. 128 p.

8. Sayyah M, Shirbandi K, Saki-Malehi A, Rahim F. < div >Use of a problem-based learning teaching model for undergraduate medical and nursing education: a systematic review and meta-analysis </div>. Adv Med Educ Pract. 2017;Volume 8:691-700.

9. Cohen-Schotanus J, Muijtjens AMM, Schönrock-Adema J, Geertsma J, Van Der Vleuten CPM. Effects of conventional and problem-based learning on clinical and general competencies and career development. Med Educ. 2008;42(3):256-65.

10. Schauber SK, Hecht M, Nouns ZM, Kuhlmey A, Dettmer $\mathrm{S}$. The role of environmental and individual characteristics in the development of student achievement: a comparison between a traditional and a problem-based-learning curriculum. Adv Heal Sci Educ. 2015;20(4):1033-52.

11. Fatmi M, Hartling L, Hillier T, Campbell S, Oswald AE. The effectiveness of team-based learning on learning outcomes in health professions education: BEME Guide No. 30. Med Teach. dezembro de 2013;35(12):e1608-24.

12. Dolmans D. The effectiveness of PBL: the debate continues. Some concerns about the BEME movement. Med Educ. dezembro de 2003;37(12):1129-30.
13. Slavin SJ, Schindler DL, Chibnall JT. Medical student mental health 3.0: Improving student wellness through curricular changes. Acad Med. 2014;89(4):573-7.

14. Jha V, McLean M, Gibbs TJ, Sandars J. Medical professionalism across cultures: A challenge for medicine and medical education. Med Teach. 2015;37(1):74-80.

15. Norman GR, Schmidt HG. Revisiting 'effectiveness of problem-based learning curricula: theory, practice and paper darts'. Med Educ. 2016;50:793-7.

16. Gijbels D, Dochy F, Van den Bossche P, Segers M. Effects of Problem-Based Learning: A Meta-Analysis From the Angle of Assessment. Rev Educ Res. março de 2005;75(1):27-61.

17. Haddad AE, Ristoff D, Passarella TM. A Aderência dos Cursos de Graduação em Enfermagem , Medicina e Odontologia às Diretrizes Curriculares Nacionais. Ministério da Saúde, organizador. Ministério da Saúde Ministerio da Educação. Brasília (DF); 2006. 162 p.

18. García-Bellido, R.; González Such, J. y Jornet Meliá JM. Alfa De Cronbach. Encontro da Associação Nacional de Pós-graduação e Pesquisa em Administração. 2010. p. 2-4.

19. Matthiensen A. Uso do Coeficiente Alfa de Cronbach em Avaliações por Questionários. Publicações da Embrapa Roraima 2011 p. 1-31.

20. Frias D. Alfa de Cronbach y consistencia interna de los ítems de un instrumento de medida. Univ Val. 2006;(2003):1-3.

21. Maroco J, Garcia-Marques T. Qual a fiabilidade do alfa de Cronbach? Questões antigas e soluções modernas? Laboratório Psicol. 2013;4(1).

22. Huang G, Paes ÂT. Por dentro da estatística Posso usar o teste $\mathrm{t}$ de Student quando preciso comparar três ou mais grupos? Educ Contin Saúde. 2009;7(2):63-4.

23. Stella RC de R, Puccini RF. A formação profissional no contexto das Diretrizes Curriculares Nacionais para o curso de medicina. A formação médica na Unifesp Excel e compromisso Soc [online]. 2008;53-69.

24. Silva LR. UNESCO: os quatro pilares da "Educação pósmoderna. Inter-Ação Rev Fac Educ. 2008;33(2):359-78.

25. SINAES. Instrumento de Avaliação Institucional Externa. Bras Ministério da Educ. 2014;36.

26. Frank JR, Jabbour M, Fréchette D, Marks M, Valk N, Bourgeois G. The CanMEDS 2005 Physician Competency Framework. Better standards. Better physicians. Better care. Framework. 2005. 1-40 p.

27. Davis MH, Harden RM. Planning and implementing an undergraduate medical curriculum: The lessons learned. Med Teach. 3 de novembro de 2003;25(6):596-608.

28. Hiss RG, Peirce JC. A strategy for developing educational objectives in medicine: Problem-solving skills. Vol. 49, Journal of Medical Education. 1974. p. 660-5. 
29. General Medical Council. Tomorrow's Doctors: outcomes and standards for undergraduate medical education. Manchester, UK: General Medical Council; 2016. 100 p.

30. Association of American Medical Colleges and the American Medical Association.The Liaison Committee on Medical Education. Functions and structure of a Medical School. Washington, DC: Association of American Medical Colleges and the American Medical Association; 2018. 35 p.

\section{CONTRIBUIÇÃO DOS AUTORES}

Carlos Alberto de Oliveira, pesquisador principal, participou como juiz na análise de conteúdo dos projetos pedagógicos dos cursos, das diretrizes curriculares dos cursos de Medicina e das dimensões de alinhamento. Responsável pela escrita e análises. Eliana Amaral, orientadora do doutorado (Programa de Clínica Médica - área de concentração Ensino em Saúde - Unicamp), contribuiu com a elaboração do projeto e desenho do estudo, análise dos dados e preparação do texto.
Maria Helena Senger, pesquisadora associada, participou como juíza na análise de conteúdo dos projetos pedagógicos dos cursos, das diretrizes curriculares dos cursos de Medicina e das dimensões de alinhamento e participou da revisão do texto. Oscarina da Silva Ezequiel, pesquisadora colaboradora, participou como juíza na análise de conteúdo dos projetos pedagógicos dos cursos, das diretrizes curriculares dos cursos de Medicina e das dimensões de alinhamento, da revisão do texto, análise dos dados e redação final do texto.

\section{CONFLITO DE INTERESSES}

Os autores declaram não ter conflito de interesses.

\section{ENDEREÇO PARA CORRESPONDÊNCIA}

Carlos Alberto de Oliveira

Alameda dos Beija Flores 296 Q22 L10

13308-601. City Castelo - ITU - SP 